\title{
Frecuencia de Obesidad y Sobrepeso en una Institución de Salud de la Ciudad de Barranquilla
}

\section{Prevalence of Overweight and Obesity in a Health Institution from Barranquilla}

\author{
Mirary Mantilla Morrón ${ }^{1}$ \\ Universidad Simón Bolívar
}

\section{RESUMEN}

Introducción: Por su relación con la alta frecuencia de enfermedades crónicas no transmisibles, el sobrepeso y la obesidad se han convertido en un problema emergente de salud pública. Objetivo: Determinar la prevalencia de sobrepeso y obesidad en los sujetos afiliados a una institución de salud en la ciudad de Barranquilla. Materiales y métodos: Se realizó un estudio transversal en 245 personas que asisten a una institución de salud de primer nivel, ubicada en la ciudad de Barranquilla. Como indicador de sobrepeso y obesidad, se utilizó el índice de masa corporal (IMC), definido mediante la medición individual de peso y talla. Además, se aplicó una encuesta para identificar las variables sociodemográficas de los sujetos de estudio. Resultados: La frecuencia de sobrepeso fue del $77,9 \%$ para mujeres, y $22,1 \%$ para los hombres. La de obesidad fue de un $82,8 \%$ para las mujeres, y $17,2 \%$ para los hombres. La frecuencia de sobrepeso y obesidad aumentó específicamente con la edad tanto en varones como en mujeres, observándose las proporciones del IMC más elevadas en los mayores de 55 años, así: 35,1\% en sobrepeso, y 23,7\% en obesidad. Conclusión: El sobrepeso y la obesidad son factores de riesgo cardiovascular presentes en la población estudiada y su proporción varía según el sexo y edad de las personas.

Palabras Clave: obesidad, sobrepeso, edad, sexo (Fuente: DeCS).

\begin{abstract}
Introduction: Obesity and overweight have become an emerging public health problem, by its relationship with the high frequency of chronic non-communicable diseases. Objective: To determine the prevalence of overweight and obesity in subjects affiliated with an institution of health in Barranquilla. Materials and methods: study of 245 people who attend an institution of first level health located in Barranquilla. (BMI) body mass index, defined by individual measuring weight and height, is used as an indicator of obesity. In addition a survey was applied to identify the socio-demographic variables of the study subjects. Results: the frequency of overweight was $77.9 \%$ for women and $22.1 \%$ for men and obesity was $82.8 \%$ for women and $17.2 \%$ for men. Overweight and obesity increased specifically with age in males and females, noting the proportions of BMI higher in people over 55 years, $35.1 \%$ overweight, $23.7 \%$ in obesity. Conclusions: overweight and obesity are cardiovascular risk factors present in the studied population, and their proportion varies according to sex and age of the people.
\end{abstract}

Keywords: obesity, overweight, age, sex (Source: MeSH, NLM). 


\section{Introducción}

La El sobrepeso y la obesidad se han convertido en un grave problema de salud pública (1). En su etiología se reconocen factores genéticos y estilos de vida no saludables (2). Como ha ocurrido en el mundo desarrollado y en muchos países en vía de desarrollo, en Colombia, este problema se ha incrementado de forma alarmante, en particular, en los entornos urbanos, afectando no solo a la población adulta, sino a niños y jóvenes (3).

La obesidad se ha constituido así en el principal problema de malnutrición en el adulto, y aun se hacen notorios en la población infantil (4). Según la Organización Mundial de la Salud (OMS) y el grupo Internacional de Trabajo en Obesidad (IOTF), esta enfermedad es definida como acumulación anormal y excesiva de grasa que pone en riesgo la salud. Por su impacto sobre la morbilidad y mortalidad, la calidad de vida y el gasto sanitario, ella es considerada la epidemia de siglo XXI (5).

Además, la obesidad es calificada como el quinto factor de riesgo de muerte en el mundo, así como de una serie de enfermedades crónicas, incluyendo diabetes, enfermedades cardiovasculares y cáncer (6). De acuerdo con los últimos datos, más de un billón de adultos padecen sobrepeso y 300 millones de ellos son obesos (7). Cada año fallecen como mínimo 2,8 millones de personas a causa del sobrepeso y la obesidad. Asimismo, el 44\% de la carga de diabetes, el $23 \%$ de las cardiopatías isquémicas y entre el $7 \%$ y el $41 \%$ de la carga de algunos cánceres se le atribuyen al sobrepeso y a la obesidad. En 2008, 1500 adultos mayores de 20 años padecían sobrepeso, de los cuales 200 millones eran hombres y 300 millones, mujeres. En 2010, aproximadamente 43 millones de niños menores de 5 años de edad tenían sobrepeso, de los cuales, cerca de 35 millones vivíanen países en desarrollo, mientras que en los países desarrollados esa cifra era de 8 millones (8).

Entre1980 y 2008, la prevalencia mundial de sobrepeso y obesidad se ha duplicado (9). Y, aunque existen importantes diferencias entre países, el trabajo realizado por expertos del Imperial College de Londres (Reino Unido), y de la facultad de Salud pública de la Universidad de Harvard (Estados Unidos) concluye que la obesidad, al igual que el colesterol o la hipertensión, ya no son únicamente problemas de Occidente o de las naciones ricas, ahora, estos trastornos están presentes también en los países de medianos y bajos ingresos (10). Se trata, entonces, de una amenaza global para la salud pública. En 2008, el $10 \%$ de los hombres y el $14 \%$ de las mujeres del mundo eran obesos (IMC $\geq 30 \mathrm{~kg} / \mathrm{m} 2$ ), en comparación con el 5\% para los hombres y el $8 \%$ de las mujeres en 1980 (11).

La prevalencia del sobrepeso y la obesidad fueron más altas en la región de América (62\% de sobrepeso y el $25 \%$ de obesidad en ambos sexos), y la menor en el sudeste de Asia (14\% de sobrepeso en ambos sexos y el $3 \%$ para la obesidad). La frecuencia del elevado índice de masa corporal aumenta con el nivel de ingresos de los países, desde los niveles máximos hasta los ingresos medios (12).

Según la encuesta Nacional de Situación Nutricional en Colombia (ENSIN), en 2010 casi la mitad de los colombianos sufrían de sobrepeso y obesidad, alcanzando niveles alarmantes. Pero la incidencia del problema puede ser mayor: el porcentaje de mujeres entre 19 y 64 años de edad que presentan sobrepeso y obesidad es de un $55,2 \%$, mientras que en los hombres es de 45,6\%. El indicador mayor se observa en los niveles socioeconómicos más bajos y cuando sólo han cursado niveles de primaria (13). En el orden departamental, San Andrés y Providencia, Guaviare, Guainía, Vichada y Caquetá presentan las mayores prevalencias, todas superiores al 57\% del total de su población (13). En el sur del departamento del Atlántico, más de un $70 \%$ de la población presentó sobrepeso y obesidad, observándose medidas antropométricas más elevadas en las mujeres que en los hombres (14).

De otra parte, al menos 18 comorbilidades se asocian con el exceso de peso, y los riesgos relativos son algo diferentes entre hombres y mujeres, y estos varían según el género y la edad y se agravan con el exceso de peso (15). En forma más concreta, el sobrepeso y la obesidad en los hombres conlleva un mayor riesgo que de sufrir cáncer colon rectal, derrame cerebral y artrosis (16); mientras que las mujeres con sobrepeso sufren mayor riesgo de padecer diabetes, enfermedades del corazón, endometriosis y cáncer en ovarios (17).

Por otro lado, el riesgo de enfermedad cardiovascular se incrementa con la edad. Para los hombres, el riesgo de aumento inicia a partir de los 45 años, encontrándose que 10 de cada 1000 hombres 
desarrollan signos de enfermedad cardiaca. Al llegar a los 55 años, el riesgo se ha duplicado a 21 de cada 1000 hombres, y este sigue aumentando hasta que, alrededor de los 85 años, 74 de cada 1000 hombres padecen la enfermedad cardiaca (18).

En las mujeres, el riesgo de enfermedad cardiovascular también aumenta con la edad, aunque en ellas suele aparecer 10 años más tarde que en los hombres $\mathrm{y}$, especialmente, tras la aparición de la menopausia (19). En síntesis, los hombres y mujeres mayores de 65 años tienen mayor probabilidad de morir por un infarto al miocardio que los jóvenes (20).

Mantener el adecuado peso corporal es un determinante principal para la supervivencia de la especie humana. Pero, para mantener un equilibrio entre el peso corporal y la constitución del cuerpo se requiere que el consumo y el gasto de energía sean inversamente proporcionales (21), pues, justamente, el desequilibrio crónico entre la ingesta de energía y el gasto de energía da como resultado cambios en la masa del tejido adiposo (22).

En todo caso, no se debe olvidar que el sobrepeso y la obesidad constituyen un problema de salud multifactorial: la influencia de la cultura, el nivel social, la genética, el sexo y la edad pueden explicar su presencia. Los agentes ambientales también tienen una importancia considerable en este desequilibrio. Así, en un ambiente obesicogénico, el factor genético implicado en la obesidad regula la distribución de la grasa corporal, el ritmo del metabolismo, la respuesta al ejercicio físico, la dieta, el control del pensamiento y la preferencia por los alimentos (23). Además, el proceso de modernización y reestructuración socioeconómica de los países en desarrollo y en vía de desarrollo han modificado sus estilos de vida, afectando con ello los modelos nutricionales como la ingesta de calorías de origen animal y grasas saturadas, a lo que se suman los cambios de los hábitos sociales y la reducción de la actividad física (24).

Se requiere, en consecuencia, un enfoque preventivo global, que incluya la familia, la escuela y las normas de salud pública a nivel local, regional y mundial, para promover un estilo de vida saludable con dietas y actividad física (25). En nuestro caso, dada la importancia de estudiar la problemática de la obesidad en las poblaciones para abordar el problema, este artículo tiene como objetivo determinar la frecuencia de sobrepeso y obesidad en un grupo de personas que asisten a una institución de salud de primer nivel de la ciudad de Barranquilla.

\section{Materiales y Métodos}

Se realizó un estudio descriptivo transversal en 245 personas de ambos sexos, que asisten a una institución prestadora de salud en la ciudad de Barranquilla.

Se seleccionaron a todas las personas que asistieron durante los meses de agosto y septiembre de 2011 a la consulta de promoción de la salud de dicha entidad. Para la medición del peso, se utilizó una balanza marca Tanita, y la talla se obtuvo mediante un tallímetro. En ambas mediciones, se usaron los procedimientos estandarizados $\mathrm{y}$ avalados por organizaciones internacionales (18). Al medir la talla, el participante se presentó sin zapatos u objetos sobre la cabeza, los pies juntos, los talones contra el tallímetro, las rodillas rectas y en inspiración. Para medir el peso, se le pidió que subiera a la balanza sin zapatos, que no se moviera, que mirara hacia adelante y mantuviera los brazos a cada lado de su cuerpo.

Con base en la talla y el peso, se definió el índice de masa corporal, considerando la presencia de obesidad cuando los valores de IMC eran iguales o superiores a $30 \mathrm{~kg} / \mathrm{m} 2$, y en el caso del sobrepeso, se consideraron estándares iguales o superiores a $25-30 \mathrm{~kg} / \mathrm{m} 2$.

La información de las variables sociodemográficas se recolectó a través de una encuesta, previo consentimiento informado. El análisis de los datos se realizó mediante el paquete estadístico SPSS 18, el cual permitió estimar las medidas descriptivas y de tendencia central. Para estimar las relaciones entre el IMC, el sexo y el rango de edad de los participantes, se usó la prueba de $\mathrm{Chi}^{2}$.

\section{Resultados}

El 78\%de los encuestados fueron mujeres y todos los sujetos pertenecen al estrato socioeconómico 1. Además, el $35,1 \%$ tenía sobrepeso y el $23,7 \%$ obesidad, como lo muestra la Tabla 1. 
Tabla 1. Características sociodemográficas y antropométricas de los participantes

\begin{tabular}{lcc}
\hline \multicolumn{1}{l}{} & Frecuencia & Porcentaje \\
\hline Sexo & & \\
\hline Femenino & 191 & $78,0 \%$ \\
Masculino & 54 & $22,0 \%$ \\
\hline Estrato socioeconómico & & \\
\hline 1 & 245 & $100,0 \%$ \\
\hline \multicolumn{1}{l}{ Índice de masa corporal } & & \\
\hline Delgado & 14 & $5,7 \%$ \\
Normal & 87 & $35,5 \%$ \\
Sobrepeso & 86 & $35,1 \%$ \\
Obesidad & 58 & $23,7 \%$ \\
\hline
\end{tabular}

En la Tabla 2 se presentan las medias y la desviación estándar de las variables cuantitativas que se consideraron de mayor interés: edad e IMC.

Tabla 2. Medias de edad e índice de masa corporal

\begin{tabular}{lccc}
\hline \multicolumn{1}{c}{ Variables } & Media & $\begin{array}{c}\text { Desviación } \\
\text { Estandar }\end{array}$ & Rango \\
\hline $\begin{array}{l}\text { Edad } \\
\text { Índice de masa } \\
\text { corporal }\end{array}$ & 61,9 & 16,7 & $11-63$ \\
\hline
\end{tabular}

La Figura 1 muestra las frecuencias del IMC de hombres y mujeres, observándose prevalencias mayores en las mujeres.

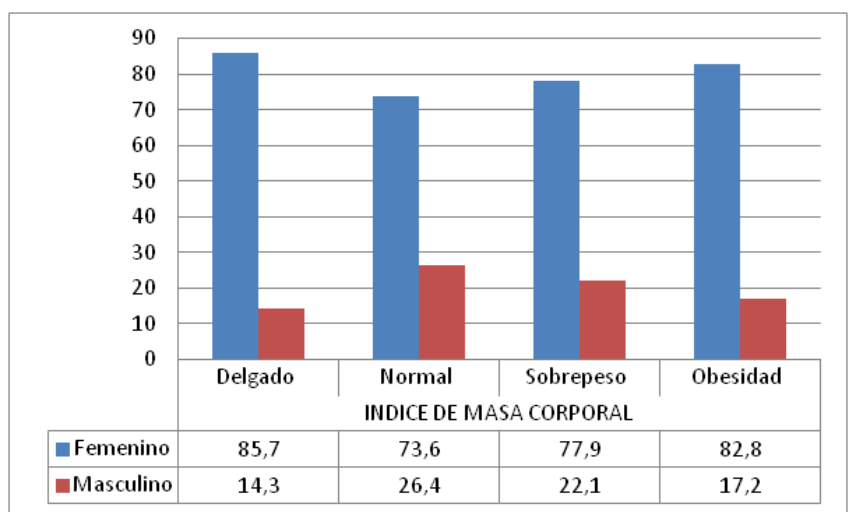

Figura 1. Índice de masa corporal según el sexo de los sujetos

En la Tabla 3, se observa la relación entre el índice de masa corporal y el rango de edad de los sujetos, hallando diferencias significativas $(\mathrm{p}=0,0001)$, evidenciándose que las personas mayores tienen una mayor prevalencia de sobrepeso y obesidad.

Tabla 3. Índice de masa corporal de acuerdo con el rango de edad

\begin{tabular}{ccc}
\hline Rango de Edad & Delgado / Normal & $\begin{array}{c}\text { Sobrepeso / } \\
\text { Obesidad }\end{array}$ \\
\hline $11-17$ & $1(0,7)$ & $7(6,9)$ \\
$18-29$ & $3(2,1)$ & $1(1,0)$ \\
$30-49$ & $32(22,2)$ & $5(5,0)$ \\
$50-65$ & $51(35,4)$ & $29(28,7)$ \\
Mayor de 65 & $57(39,6)$ & $59(58,4)$ \\
\hline * $=0,0001$
\end{tabular}

\section{Discusión}

En el presente estudio, se encontraron prevalencias de sobrepeso y obesidad en hombres y mujeres con un valor de $35.1 \%$ y $23.7 \%$, respectivamente. $\mathrm{Al}$ analizar los datos obtenidos, se halló que estos fueron similares a los reportados por Cea-Calvo en España (26). También son comparables con los de la Encuesta Nacional de Situación Nutricional (ENSIN) (13), según la cual la población colombiana presentó una prevalencia de $34.6 \%$ en sobrepeso, y de $16.5 \%$ en obesidad.

El principal hallazgo de este estudio consistió en una tendencia ascendente de sobrepeso y obesidad en relación directa con el incremento de la edad, sobre todo en el sexo femenino. $\mathrm{Su}$ prevalencia ha sido similar a la obtenida por otros autores nacionales y extranjeros $(27,28)$, quienes manejan cifras que oscilan entre el $20 \%$ y el $30 \%$ de la población, tanto en países desarrollados como en vía de desarrollo.

Asimismo, nuestros resultados son semejantes a los encontrados por Manzur (29) y Martínez (30), para quienes la proporción de sujetos con un IMC elevado fue mayor en las mujeres. Además, la mayoría de los sujetos presentan uno o más factores de riesgo cardiovascular en comparación con los sujetos que presentan un IMC normal (31). Gonzales encontró al respecto una mayor prevalencia de obesidad en niñas de 12 y 13 años, mientras que en los niños la prevalencia fue inferior hasta los 13 años. Estos últimos mostraron un incremento en la obesidad a partir de esta edad, encontrándose una correlación significativa entre el IMC y la edad (32). 
Por su parte, Flegal (33) evaluó la relación de la obesidad en mujeres y hombres según el IMC y edad, observando que, en un periodo de los 12 años, la prevalencia de la obesidad ajustado a la edad no mostró aumento significativo entre las mujeres en general, pero sí fueron representativos en la raza hispana y negra. Del mismo modo, en los hombres se manifestó una tendencia lineal significativa y las tendencias del IMC fueron similares a las de la obesidad.

Yatsuya (34) concluyó en su estudio que el grado de obesidad definido por el IMC es un importante factor de riesgo para accidente cerebro-vascular, independientemente del genero o de la raza. De todos modos, en su análisis, los negros tenían aproximadamente de 2 a 3 veces más altas tasas de incidencia de enfermedad cerebro-vascular isquémico en comparación con los blancos en cada quintil de la obesidad. Con base en la estimación del IMC, Vemmos (35), por su parte, observó que las tasas de supervivencia en pacientes con enfermedad cerebrovascular y exceso de peso fueron significativamente mayores que en los pacientes con un IMC normal.

Ahora bien, los resultados del presente estudio son opuestos a lo descrito por Rodríguez (36), quien encontró que la prevalencia de sobrepeso fue mayor en los hombres, mientras que en la obesidad no existió diferencia entre sexos. En los países en vía de desarrollo, la prevalencia de obesidad también suele ser superior en el grupo femenino, mientras que en los países desarrollados es variable y depende de las características de cada sujeto (37). De acuerdo con Flegal (33), en Estados Unidos, por ejemplo, la prevalencia de sobrepeso y obesidad en hombres y mujeres es similar: $35,5 \%$ para los hombres, y 35,8\% para las mujeres y no se han presentado cambios significativos desde 2003 a 2008.

En definitiva, nuestros resultados permiten concluir que la frecuencia de sobrepeso y obesidad coincide con resultados nacionales e internacionales. Igualmente, obligan a los profesionales de la salud a diseñar e implementar programas de intervenciones interdisciplinarias y multifactoriales, que conlleven a la disminución de esta problemática de salud pública.

\section{Referencias}

1. Álvarez DD, Sánchez AJ, Gómez GG, Tarqui MC. Sobrepeso y Obesidad: Prevalencia y Determinantes Sociales del Exceso de Peso en la Población Peruana (2009-2010). Rev Perú Med Exp Salud Pública. 2012; 29(3):303-13.

2. Braguinsky J. Prevalencia de Obesidad en América Látina. Anales Sis San Navarra. 2002; 25 (1): 109-115.

3. Alvarez A, Gehrig P A. la obesidad: un problema demasiado grande para ignorarlo. Oncología Ginecológica. 2012; 126 (2):274-276.

4. Achor MS, Benites CN, Brac ES, Barslund SA. Obesidad infantil. Rev Posgrado VI Cátedra de Medicina. 2007; 168:34-38.

5. World Health Organization. Obesidad y sobrepeso. Nota descriptive $N^{\circ}$ 311. Mayo 2012. Disponible en: http://www.who.int/mediacentre/factsheets/fs311/ es/index.html

6. Morales A, Coca A. Obesidad, actividad física y riesgo cardiovascular: Clasificación ergoantropométrica, variables farmacológicas, biomarcadores y "paradoja del obeso". Med Clin. 2010; 134 (11): 134-492.

7. Hansen JC, Gilman AP, Odland JO. Is thermogenesis a significant causal factor in preventing the "globesity" epidemic? Medical Hypotheses. 2010; 75(2): 250-256.

8. Lantigua IF. El mapa global de la Obesidad. Evolución del Índice de masa corporal. Febrero 2011. Disponible en: http://www.elmundo.es/elmundosalud/2011/02/03 /nutricion/1296763533.html

9. Ruiz L, Zapico M, Zubiaur A, Sanchez J, Florez J. Aumento de la prevalencia de sobrepeso y obesidad en la población infantil de la provincia de Alicante en los últimos 10 años. Endocrinol Nutr. 2008; 55 (09):389-395.

10. Roccisano D, Henneberg M. Soy consumption and obesity. Food and Nutrition Sciences. 2012; 3: 260-266.

11. Laslett LJ, Alagona P, Clark BA, Drozda JP, Saldevar F, Wilson SR, et al. Worldwide environment of cardiovascular disease: 
prevalence, diagnosis, therapy, and policy issues a report from the American College of Cardiology. Journal of the American College of Cardiology. 2012; 60(25): 1-49.

12. Chan RS, Woo J. Prevención del sobrepeso y la obesidad: ¿Que tan eficaz es el enfoque actual de la salud pública? Int $\mathrm{J}$ Environ Res Public Health. 2010; 7 (3): 765-783.

13. Instituto de Bienestar familiar. Encuesta Nacional de la Situación Nutricional en Colombia 2010.ENSIN. 2 Edición. 2010.

14. Navarro E, Vargas R. Síndrome metabólico en el suroccidente de Barranquilla (Colombia). Salud Uninorte. 2008; 28 (1): 49-64.

15. Vásquez $M$, Ulate G. Regulación del peso corporal y del apetito. Acta méd. Costarric. 2010; 52 (2): 79-86.

16. Guh DP, Zhang W, Bansback N, Amarsi Z, Birmingham CL, Anis AH. The incidente of comorbidities related to obesity and overweigth: a systematic review and meta-analysis. BMC Public Health. 2009; 9(88):1-20.

17. Osorio O, Amaya MC. Overview of feeding practices in school adolescents. Avances en Enfermería. 2010; 27(2): 43-56.

18. Daniilidis A, Dinas K. Long term health consequences of polycystic ovarian syndrome: a review analysis. Hippokratia. 2009; 13(2): 90-92.

19. Andersen K, Andersen ZJ, Olsen TS. Age and gender-specific prevalence of cardiovascular risk factors in 40102 patients with first-everischemic. Stroke. 2010; 41: 2768-2774.

20. Towfighi A, Zheng L, Ovbiagele B. Weight of the obesity epidemicrising Stroke rates among middle-aged women in the United States. Stroke. 2010; 41:1371-1375.

21. Ley R E, Obesity and the human microbiome. Current opinion in astroenterology 2010;26:5-11

22. Pinheiro AC, Canaan FA, Goncalves RD. Insulemia, ingesta alimentaria y metabolismo energético. Rev. chil. nutr. 2008; 35 (1):18-24.

23. Hebebrand J, Hinney A. Environmental and genetic risk factors in obesity. Child Adolesc Psychiatr Clin N Am. 2009;18(1):83-94.
24. Morales M, Carvajal CF. Obesidad y resistencia a la leptina. Gac Med Bol. 2010; 33 (1):63-68.

25. Luckie A, Cortés F, Ibarra S. Obesidad: transcendencia y repercusión médico social. Revista de especialidades Médico- Quirúrgicas. 2009; 14 (4):191-201.

26. Cea CL, Moreno B, Monereo S, Gil GV, Lozano JV, Martí JC, et al. En representación de los investigadores del estudio PREV-ICTUS. Medicina Clínica. 2008; 131 (6): 205-210.

27. Reyes JM, Díaz BE, Lera ML, Burrows AR. Ingesta y metabolismo energético en una muestra de adolescentes chilenos con sobrepeso y obesidad. Rev. méd. 2011; 139(4): 425-431.

28. Márquez AJ, García VV, Ardila CR. Ejercicio y prevención de obesidad y diabetes mellitus gestacional. Rev. chil. obstet. ginecol. 2012; 77(5): 401-406.

29. Manzur F, De la Ossa M, Trespalacios E, Abuabara Y, Luján M. Prevalencia de sín-drome metabólico en el municipio de Arjo $\neg$ na, Colombia. Revista Colombiana de Cardio $\neg$ logía. 2008; 15 (5): 215-222.

30. Martínez S, Romero P, Ferri J, Pedro T, Real J, Priego A, et al. Perímetro de cintura y factores de riesgo cardiovascular. Revista Española de Obesidad. 2008; 6 (2): 97-104.

31. Liu K, Daviglus ML, Loria CM, Colangelo LA, Spring B, Moller AC, et al. Healthy lifestyle through young adulthood and the presence of low cardiovascular disease risk profile in middle age. The coronary artery risk development in (young) adults (CARDIA) study. Circulation. 2012; 125: 996-1004.

32. González E, Aguilar MJ, García CD, García PA, Padilla CA, Álvarez J. Estudio epidemiológico de enfermería sobre la prevalencia de sobrepeso, obesidad y su asociación con hipertensión arterial en una población de estudiantes en la ciudad de Granada y su provincia. Nutr. clín. diet. hosp. 2010; 30(2):42-50.

33. Flegal KM, Carroll MD, Kit BK, Ogden CL. Prevalence of obesity and trends in the distribution of body mass index among US adults, 1999-2010. JAMA. 2012; 307(5):491-497. 
34. Yatsuya H, Folsom AR, Yamagishi K, North KE, Brancati FL, Stevens J. Race-and sex-specific associations of obesity measures with ischemic stroke incidence in the atherosclerosis risk in communities (ARIC) Study. Stroke. 2010; 41:417-425.

35. Vemmos K, Ntaios G, Spengos K, Savvari P, Vemmou A, Pappa T, et al. Association between obesity and mortality after acute first-ever stroke: the obesity-stroke paradox. Stroke. 2011; 42(1):30-36.

36. Rodríguez RE, López PB, López SA, Ortega RM. Prevalencia de sobrepeso y obesidad en adultos españoles. Nutr. Hosp. 2011; 26(2): 355-363.

37. Téstar DJ, Guedes R, Madruga C, León L, Posada PR, Laureiro I. Obesidad, su relación con otros factores de riesgo coronario. Rev. Med. Electrón. 2009; 31(6): 1-29. 